\title{
Multiple vegetative lesions revealing superficial granulomatous pyoderma
}

\author{
Fatima Azzahra El Gaitibi', Sanae Sialiti', Mehdi Khalaayoune', Kaoutar Znati², \\ Mariame Meziane ${ }^{1}$, Nadia Ismaili' ${ }^{1}$, Leila Benzekri ${ }^{1}$, Karima Senouci ${ }^{1}$
}

${ }^{1}$ Department of Dermatology, Mohammed V University in Rabat, Ibn Sina University Hospital, Rabat. Morocco, ${ }^{2}$ Department of Histopathology. Mohammed V University in Rabat, Ibn Sina University Hospital, Rabat. Morocco.

Corresponding author: Fatima Azzahra El Gaitibi, MD, E-mail: elgaitibi.fatimaazzahra@gmail.com

Sir,

First described by Wilson-Jones and Winkelmann in 1988 [1], superficial granulomatous pyoderma (SGP) is a rare disease that is considered a variant of pyoderma gangrenosum. SGP differs from the classic form by its indolent clinical course, a lack of an associated and underlying disease, the presence of a granulomatous infiltrate on histology, and a better prognosis with less aggressive therapies [2].

A sixty-year-old male with no known past medical history presented himself with multiple verrucous skin lesions. These started eight months earlier as nodules and rapidly progressed into vegetating purplish erythematous plaques gradually increasing in size, localized on the abdomen, pubic area, and buttocks. A clinical examination revealed crusty and purplish verrucous plaques on the abdomen (Fig. 1), vegetative nodular lesions in the pubic area (Fig. 2), and an ulcerative painful lesion on the buttocks (Fig. 3). There was also a cribriform scar on the left shoulder. Besides, the patient had no systemic symptoms and otherwise felt well. The first biopsy revealed a neutrophilic infiltrate suggestive of an infectious origin. The patient was treated with antibiotics for fifteen days without an improvement. A second biopsy was performed, along with histological, bacteriological, mycological, and parasitological studies. Histology revealed pseudoepitheliomatous hyperplasia, a polynuclear neutrophilic infiltrate with eosinophils, and focal vasculitic lesions (Fig. 4). Mycological, bacteriological, and parasitological examinations were unremarkable. In light of this, the main differential diagnoses were excluded: vegetative tuberculosis, verrucous leishmaniasis, and deep mycosis. Syphilitic serology was unremarkable. Eventually, the diagnosis of superficial granulomatous pyoderma was made. Complete blood count, serum protein electrophoresis, and paraneoplastic workup were normal. Colonoscopy was performed, ruling out Crohn's disease. Systemic corticosteroid therapy was initiated at a dose of $1 \mathrm{mg} / \mathrm{kg} / \mathrm{day}$, but, in the absence of improvement after one month, dapsone was introduced at a dose of $150 \mathrm{mg} /$ day with favorable progress and good tolerance.

Superficial granulomatous pyoderma (SPG) is a rare variant of granulomatous pyoderma, differing from its classic form by its painless nature, its clinical and histological appearance, and, most often, the absence of an associated pathology [3]. SGP appears as a superficial ulceration with a clean base and vegetative edges, most often single, painless, and localized at the level of the trunk or the limbs. Histology reveals a granuloma with three zones: an innermost zone of necrotic debris and neutrophils, surrounded by histiocytes and giant cells, and, lastly, encircled by plasma cells and eosinophils [2]. Other histological features include pseudo-carcinomatous hyperplasia, focal hemorrhages, vasculitic lesions, and a neutrophil infiltrate [4]. The main differential diagnoses are infectious bacterial, mycobacterial, and parasitic etiologies. The evolution of SGP is slower than the classic form and not especially aggressive and, most often, responds to simpler treatments, such as local corticosteroid therapy [4].

Our observation presents some peculiarities. The topography and the multiplicity of the lesions are

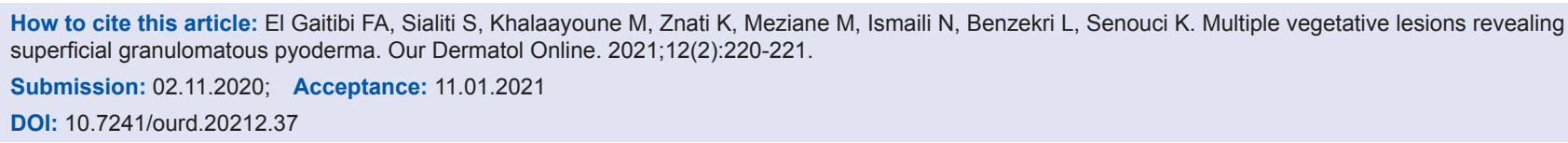




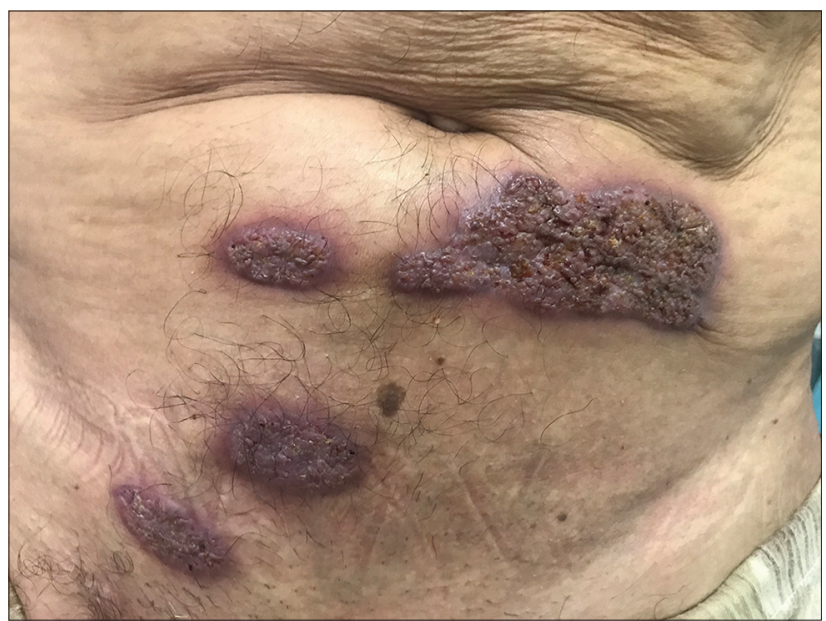

Figure 1: Crusty and purplish verrucous plaques on the abdomen.

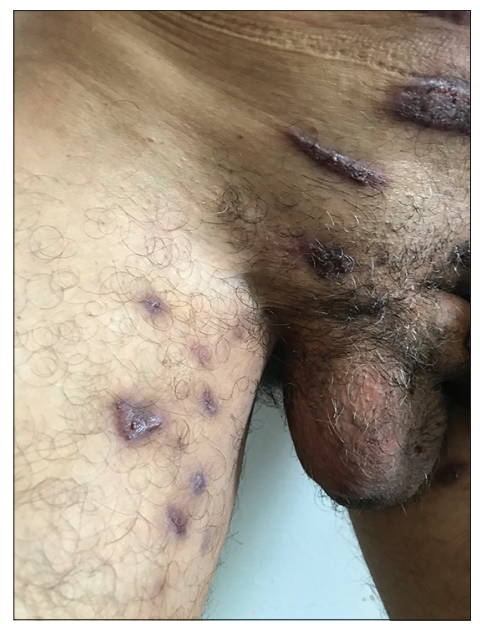

Figure 2: Multiple ulcerated nodules and plaques of the pubis and the right inguinal fold.

rarely reported in the literature [5]. In addition, the absence of a histological granuloma commonly found in SGP makes the diagnosis more difficult. The clinical and histological heterogeneity of this entity is the challenging factor faced by clinicians. SGP remains a diagnosis of exclusion based on the appearance of any vegetative lesion, evolves slowly even in the absence of a granuloma, and is composed of three histological zones.

\section{Consent}

The examination of the patient was conducted according to the principles of the Declaration of Helsinki.

The authors certify that they have obtained all appropriate patient consent forms, in which the patients gave their consent for images and other clinical information to be included in the journal. The patients understand that their names and initials will not be published and due effort will be made to conceal their identity, but that anonymity cannot be guaranteed.

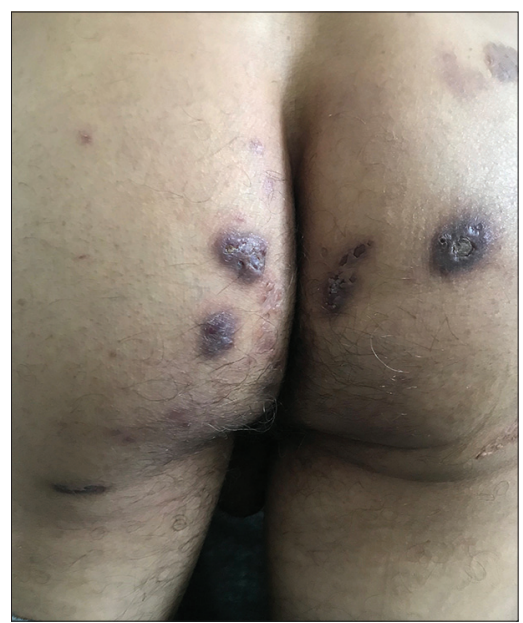

Figure 3: Multiple ulcerated purplish nodules with vegetative edges located on the buttocks.

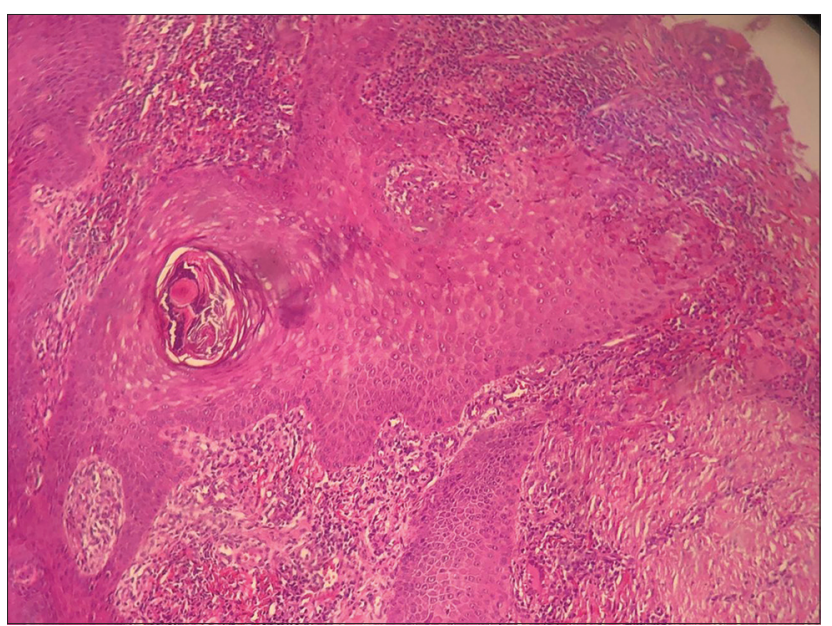

Figure 4: Histologic image showing a polynuclear neutrophilic infiltrate and focal vasculitic lesions (H\&E; 20x).

\section{REFERENCES}

1. Wilson-Jones E, Winkelmann RK. Superficial granulomatous pyoderma: A localized vegetative form of pyoderma gangrenosum. J Am Acad Dermatol. 1988;18:511-21.

2. Vasileva M, Brishkoska Boshkovski V. Pyoderma gangrenosum associated with ulcerative colitis. Case report and literature review. Our Dermatol Online. 2020;11(e):e119.1-e119.3.

3. Parker J, Liszewski W, Merten AH, Gaddis K, Pragman A, Goldfarb N. A perplexing case of superficial granulomatous pyoderma with sporotrichoidlike distribution. Dermatol Online J. 2020;26:13030/qt5173t7tx.

4. Incel Uysal P, Gur Aksoy G, Yalcin B. Clinical findings and outcomes in patients with pyoderma gangrenosum: a single tertiary centre experience. Our Dermatol Online. 2019;10:17-22.

5. Kumar S, Vinay K, Parsad D, Saikia UN, Kumaran MS. Superficial granulomatous pyoderma: A great mimicker. Indian J Dermatol Venereol Leprol. 2018;84:374.

Copyright by Fatima Azzahra El Gaitibi, et al. This is an open access article distributed under the terms of the Creative Commons Attribution License, which permits unrestricted use, distribution, and reproduction in any medium, provided the original author and source are credited.

Source of Support: Nil, Conflict of Interest: None declared. 